\title{
Amyotrophic lateral sclerosis viewed from a patient's perspective
}

\author{
M. Melazzini
}

Monaldi Arch Chest Dis 2007; 67: 1, 4-5.

President, Amyotrophic Lateral Sclerosis Italian Association, Novara, Italy.

Correspondence: Dr. Mario Melazzini, AISLA, Viale Roma 32, 28100 Novara - Italy; e-mail: presidenza@aisla.it.

Amyotrophic lateral sclerosis (ALS), often referred to as "Lou Gehrig's disease" or "Charcot disease" or "Motor neuron disease", is a progressive neurodegenerative disease that affects motor neurons (particularly I and II motor neurons), nerve cells in the brain and the spinal cord. Motor neurons extend from the brain to the spinal cord and from the spinal cord to muscles throughout the body. The progressive degeneration of the motor neurons in ALS eventually leads to their death. When the motor neurons die, the ability of the brain to initiate and control muscle movement is lost. With voluntary muscle action progressively affected, patients can no longer send impulses to the muscle fibres that normally result in muscle movement, and total paralysis sets in involving, in particular, the arms and legs, speech, swallowing and breathing. Yet, through it all, for the vast majority of patients, the mind remains unaffected as regards sensorial, sexual and sphincterial functions. Patients have no choice but to witness, totally impotent to do anything, the progressive and disabling loss of autonomy. Prognosis, from the time of diagnosis, may be from 2 to 5 years. In Italy, on average, 3 people are diagnosed with ALS each day, and there are an estimated 6-8 ALS patients each 100,000 inhabitants. Following the communication of the diagnosis, patients and their families generally feel left alone to their own destiny, often without any kind of suggestion about how to face daily life with ALS. It is therefore essential to identify patients' needs, and focus on developing an appropriate relation between physicians and patients, with a correct information both for patients and their families through to the time of decision-making in later stages of the disease. To ensure that patients and their families have an effective and really on-going assistance, it is necessary to create a network between physicians, health workers and social assistance departments and patients associations. It is also essential to plan medical and welfare interdisciplinary measures, carried out by a dedicated team, in order to respond to the various needs patients have to face, that follow a paradigmatic sequence that is different from all other neurological diseases. In fact the course of the disease is highly predictable with its various breathing and nutritional needs, even if the timing may vary. The model to propose for such an ALS network is, thus, that of a dedicated interdisciplinary team, which might include the following professional figures:

- Neurologist

- Pneumologist

- Gastroenterologist / surgeon

- Interventional radiologist

- ENT specialist/ Phoniatrist

- Critrical Care/Anesthesiologist

- Neuropsychologist

- Psychologist

- Psychiatrist

- Physiatrist

- Logo-therapist

- Occupational Therapist

- Specialized Nurses

- Clinical Trials Coordinator

Respiratory failure is the most critical stage of the disease, as depicted in the review by Chetta et al. in this issue of Monaldi [1]. Unfortunately patients are not well informed about this problem, and in fact some neurologists suggest that preventive non invasive ventilation (NIV) may allow both higher quality of life and survival [2].

The fact of poor information about respiratory problems generally means that patients are left to cope on their own 'in the dark' with a great feeling of fear. What is NIV? Will I choke to death if I don't agree to it? I can't clear away secretions because I can't cough: what will happen? Do I have to be tracheotomized? And if not, how will I die? What does the tracheotomy involve? Who will help me with it?, etc. These are the most common questions in patients' minds, and generally they do not receive an adequate answer. Why?

We, as physicians, fail to realize and understand patients' real needs and therefore do not suggest the best way to face them. We are not able to understand what patients' real feelings are: emotional discomfort, loss of dignity, daily impact with nutritional and respiratory problems, the need 
to be listened to. It is an absolute must that we give everybody - patients and their families - every possible appropriate form of treatment, care and support. And the best way to do this is through a comprehensive form of treatment, based on: a correct physicianpatient relationship, sharing correct information about the disease and all the problems related to the patient and their families, discussing together openly and frankly all that will occur in practical terms throughout the various stages of the disease.

\section{References}

1. Chetta A, Aiello M, Tzani P, Olivieri D. Assessment and monitoring of ventilatory function and cough efficacy in patients with amyotrophic lateral sclerosis. Monaldi Archives for Chest Diseases - Pulmonary Series 2007; 67: 43-52.

2. Piepers S, Van den Berg JP, Kalmijn S, et al. Effect of non-invasive ventilation on survival, quality of life, respiratory function and cognition: a review of the literature. Amyotroph Lateral Scler 2006; 7:195-200.

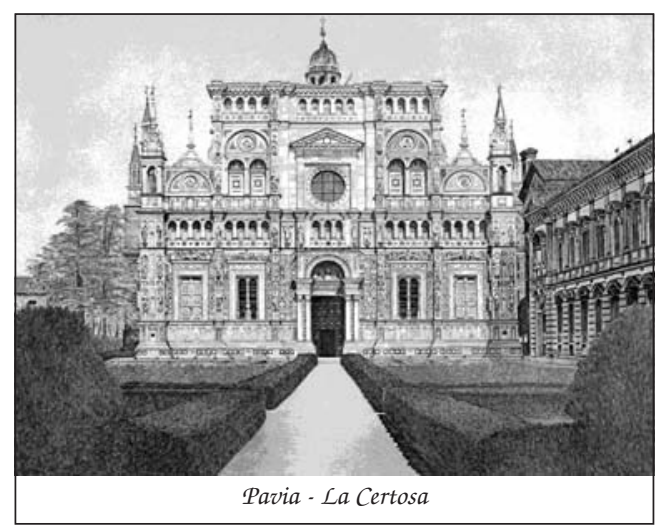

